\title{
Plaque Composition as a Predictor of Plaque Ulceration in Carotid Artery Atherosclerosis: The Plaque At RISK Study
}

(D) K. Dilba, (DD.H.K. van Dam-Nolen, (D)A.C. van Dijk, (D) M. Kassem, (D)A.F.W. van der Steen, (DP.J. Koudstaal, (DP.J. Nederkoorn, (D). Hendrikse, (DM.E. Kooi, (D).J. Wentzel, and (D) A. van der Lugt

\begin{abstract}
BACKGROUND AND PURPOSE: Plaque ulceration is a marker of previous plaque rupture. We studied the association between atherosclerotic plaque composition at baseline and plaque ulceration at baseline and follow-up.

MATERIALS AND METHODS: We included symptomatic patients with a carotid stenosis of $<70 \%$ who underwent MDCTA and MR imaging at baseline $(n=180)$. MDCTA was repeated at 2 years $(n=73)$. We assessed the presence of ulceration using MDCTA. Baseline MR imaging was used to assess the vessel wall volume and the presence and volume of plaque components (intraplaque hemorrhage, lipid-rich necrotic core, and calcifications) and the fibrous cap status. Associations at baseline were evaluated with binary logistic regression and reported with an OR and its $95 \% \mathrm{Cl}$. Simple statistical testing was performed in the follow-up analysis.

RESULTS: At baseline, the prevalence of plaque ulceration was $27 \%(49 / 180)$. Increased wall volume $(\mathrm{OR}=12.1 ; 95 \% \mathrm{Cl}, 3.5-42.0)$, higher relative lipid-rich necrotic core $(\mathrm{OR}=1.7 ; 95 \% \mathrm{Cl}, 1.3-2.2)$, higher relative intraplaque hemorrhage volume $(\mathrm{OR}=1.7 ; 95 \% \mathrm{Cl}$, $1.3-2.2)$, and a thin-or-ruptured fibrous cap $(\mathrm{OR}=3.4 ; 95 \% \mathrm{Cl}, 1.7-6.7)$ were associated with the presence of ulcerations at baseline. In $8 \%(6 / 73)$ of the patients, a new ulcer developed. Plaques with a new ulceration at follow-up had at baseline a larger wall volume $\left(1.04 \mathrm{~cm}^{3}\right.$ [IQR, $0.97-1.16 \mathrm{~cm}^{3}$ ] versus $0.86 \mathrm{~cm}^{3}$ [IQR, $\left.\left.0.73-1.00 \mathrm{~cm}^{3}\right] ; P=.029\right)$, a larger relative lipid-rich necrotic core volume (23\% [IQR, 13-31\%] versus $2 \%$ [IQR, $0-14 \%] ; P=.002)$, and a larger relative intraplaque hemorrhage volume (14\% [IQR, 8-24\%] versus $0 \%[\mathrm{IQR}, 0-5 \%] ; P<.001)$.
\end{abstract}

CONCLUSIONS: Large atherosclerotic plaques and plaques with intraplaque hemorrhage and lipid-rich necrotic cores were associated with plaque ulcerations at baseline and follow-up.

ABBREVIATIONS: AUC = area under the curve; IPH = intraplaque hemorrhage; IQR $=$ interquartile range; LRNC $=$ lipid-rich necrotic core; TRFC $=$ thick versus thin-or-ruptured fibrous cap

A therosclerosis in the carotid arteries is one of the leading causes of ischemic stroke with arterio-arterial embolism as the main mechanism. ${ }^{1,2}$ The degree of lumen stenosis and the symptomatic status of the patient are currently used for risk assessment and treatment decision-making. ${ }^{3,4}$ Patients with severe $(\geq 70 \%)$ and moderate (50\%-69\%) carotid artery stenosis benefit from carotid endarterectomy; however, the number needed to treat to prevent recurrent stroke is relatively high. ${ }^{5}$ Moreover, almost

Received March 3, 2020; accepted after revision August 9.

From the Departments of Radiology and Nuclear Medicine (K.D., D.H.K.v.D.-N., A.C.v.D., A.v.d.L.), Cardiology (K.D., A.F.W.v.d.S., J.J.W.), and Neurology (A.C.v.D., P.J.K.), Erasmus University Medical Center Rotterdam, Rotterdam, the Netherlands; Department of Radiology and Nuclear Medicine (M.K., M.E.K.), CARIM School for Cardiovascular Diseases, Maastricht University Medical Center+, Maastricht, the Netherlands; Department of Neurology (P.J.N.), University Medical Center Amsterdam, Amsterdam, the Netherlands; and Department of Radiology (J.H.), University Medical Center Utrecht, Utrecht, the Netherlands.

This research was performed within the framework of the Center for Translational Molecular Medicine (www.ctmm.nl), project PARISK (Plaque At RISK; grant 01C-202) and supported by the Dutch Heart Foundation. Kristina Dilba was supported, in part, by Toegepaste en Technische Wetenschappen, project No. 10813. half of neurologic events occur in patients with a low degree of stenosis. ${ }^{6,7}$ This finding has triggered investigations into other markers that may help to identify patients with a high risk of recurrent stroke. Much attention has been paid to markers of atherosclerosis, like plaque composition and plaque ulceration, with the aim of identifying vulnerable plaques. ${ }^{8}$ These vulnerable plaques have a high risk of rupture, which results in thrombus formation and embolization of plaque material and/or thrombus migrating into the intracranial circulation, thereby causing vascular occlusion and a subsequent ischemic stroke. ${ }^{2}$

Plaque composition is predictive of future cerebrovascular events. $^{9-12}$ Atherosclerotic plaque ulceration, visible as plaque-

Please address correspondence to Aad van der Lugt, MD, PhD, Erasmus University Medical Center Rotterdam, Department of Radiology and Nuclear medicine, Room Ne 507, Dr. Molewaterplein 40, 3015 GD, Rotterdam, the Netherlands; e-mail: a.vanderlugt@erasmusmc.nl

- Indicates open access to non-subscribers at www.ajnr.org

http://dx.doi.org/10.3174/ajnr.A6868 
surface disruption, which is a marker of previous plaque rupture, is also correlated with recurrent symptoms and associated with a higher risk of ischemic stroke. ${ }^{13,14}$ However, the relation between vulnerable plaque components and plaque rupture is rarely investigated. Both plaque composition and ulceration can be assessed in vivo with different imaging modalities, but MR imaging is the best technique to assess plaque composition due to its superior soft-tissue contrast, ${ }^{15}$ whereas MDCTA exceeds MR imaging in the detection of plaque ulcerations due to its excellent spatial resolution with the possibility of multiplanar reconstruction. ${ }^{16,17}$

Most previous studies investigated the relation between plaque ulcerations and plaque features using a cross-sectional study design. Generally, it was found that intraplaque hemorrhage (IPH), large lipid-rich necrotic core (LRNC), and thinning or ruptured fibrous cap were associated with the presence of ulcerations, ${ }^{18-21}$ while the presence of calcifications was inversely related to ulcerations. ${ }^{19}$ A prospective study in asymptomatic patients with severe carotid artery stenosis revealed that LRNC volume was a predictor of new surface disruption. ${ }^{22,23}$ The aim of the current study was to investigate, in symptomatic patients with mild-to-moderate $(30 \%-69 \%)$ carotid artery stenosis, which plaque components at baseline are predictive of plaque rupture at follow-up.

\section{MATERIALS AND METHODS Study Population}

This study is a substudy of the Plaque At RISK (PARISK) study (clinicaltrials.gov NCT01208025); details of the study design have been previously described in the study-design article. ${ }^{24}$ The PARISK study is a Dutch prospective, multicenter cohort study aimed at identifying patients with mild-to-moderate carotid artery stenosis with an increased risk of recurrent stroke by noninvasive plaque imaging.

From September 2010 to December 2014, we included 240 patients with recent ( $<3$ months) TIA, amaurosis fugax, or minor stroke due to ischemia in the territory of the carotid artery and $30 \%-69 \%$ ipsilateral carotid artery stenosis who were not scheduled for carotid endarterectomy. The degree of lumen stenosis was determined with Doppler ultrasonography or MDCTA. The upper cutoff value of $69 \%$ was based on the North American Symptomatic Endarterectomy Trial (NASCET) criteria. ${ }^{25}$ The lower cutoff value was an atherosclerotic plaque with a thickness of at least $2-3 \mathrm{~mm}$, which corresponds to European Carotid Surgery Trial (ECST) stenosis of approximately $30 \%{ }^{3}$

Noninvasive plaque imaging (Doppler ultrasonography, MDCTA, MR imaging) was scheduled in all patients at baseline. The follow-up imaging was scheduled in 118 patients. For the cross-sectional analysis, we selected patients who underwent both MDCTA and MR imaging at baseline. For the prospective analysis, we selected patients who, in addition to baseline MDCTA and MR imaging, underwent MDCTA at follow-up.

The study was approved by the institutional medical ethics committees of all participating centers (Erasmus University Medical Center Rotterdam, Maastricht University Medical Center, University Medical Center Amsterdam, University Medical Center Utrecht). Written informed consent was obtained from each participant before enrollment.

\section{Cardiovascular Risk Factors at Baseline}

Body mass index was recorded. Hypertension was defined as a systolic blood pressure of $>140 \mathrm{~mm} \mathrm{Hg}$ or a diastolic blood pressure of $>90 \mathrm{~mm} \mathrm{Hg}$ with at least 15 minutes of continuous noninvasive blood pressure measurement or treatment with antihypertensive medication. Hypercholesterolemia was defined as fasting total cholesterol of $>5 \mathrm{mmol} / \mathrm{L}$ or the use of cholesterol-lowering medication. Diabetes mellitus was defined as a fasting serum glucose level of $>6.9 \mathrm{mmol} / \mathrm{L}$, a 2-hour post-load glucose level of $>11.0 \mathrm{mmol} / \mathrm{L}$, or the use of antidiabetic medication. Smoking status was assessed at the time of the TIA or ischemic stroke and divided in 2 categories: current smoker or not a current smoker.

\section{MDCTA Data Acquisition and Analysis}

We performed image acquisition using a standardized protocol, as previously described in the study-design article. ${ }^{24}$ MDCTA of the carotid artery was performed if no contraindications for MDCTA were present (glomerular filtration rate, $<60 \mathrm{~mL} / \mathrm{min}$; a documented allergy to MDCTA contrast media). All MDCTA images were transferred to a workstation equipped with dedicated 3D analysis software (syngo.via; Siemens). The multiplanar reformatting application allowed analysis of both carotid arteries in oblique, coronal, and sagittal planes.

The symptomatic artery was analyzed. The degree of lumen stenosis in the carotid bifurcation was measured according to the ECST and NASCET criteria, perpendicular to the central lumen line. ${ }^{3,6}$ Plaque surface morphology was evaluated and classified as either ulcerated or nonulcerated. Plaque ulceration was defined as an extension of contrast material of $>1 \mathrm{~mm}$ into the atherosclerotic plaque, being visible in at least 2 perpendicular planes. ${ }^{21}$ In addition, the number of ulcerations per artery was recorded.

Plaque surface morphology was evaluated by 2 trained readers at baseline (B.H. and A.C.v.D.) and follow-up (K.D. and D.H.K.v.D.-N.). The trained readers (B.H., A.C.v.D., K.D., and D.H.K.v.D.-N.) were physicians who were first trained on a training set to identify the presence of ulceration on MDCTA. They had to successfully complete this training set before they assessed the ulcerations on the PARISK dataset. ${ }^{18}$ The third observer who was consulted for consensus in case of no agreement was a neuroradiologist with $>25$ years of experience (A.v.d.L.).

Temporal changes in plaque surface morphology were also evaluated by 2 trained readers (K.D. and D.H.K.v.D.-N.) by visual comparison of baseline and follow-up images in which an ulceration was detected, and a subdivision was made among new ulceration, persistent ulceration, and healed ulceration.

Image software (National Institutes of Health) was used to quantify calcifications in the symptomatic carotid artery within $3 \mathrm{~cm}$ proximal and distal to the bifurcation. We used a threshold of $600 \mathrm{HU}$ to differentiate calcifications from contrast material in the lumen; calcification volume was expressed in cubic millimeters. ${ }^{26}$

\section{MR Imaging Data Acquisition and Analysis}

All MR imaging examinations were performed on 3T wholebody MR imaging scanners (Achieva, Philips Healthcare, or Discovery MR 750, GE Healthcare) using an 8-channel phasedarray coil (Shanghai Chenguang Medical Technologies) or a 4channel phased-array coil with an angulated setup (PACC-GS30. 
Machnet B.V.), respectively. MR imaging of the carotid artery was performed if no contraindications for MR imaging were present (claustrophobia). Imaging protocols included 5 sequences that were comparable among centers (Philips: 3D-TOF fast-field echo, 3D-T1WI inversion recovery Turbo field echo, 2D-T2WI TSE, 2D-T1WI Quadruple inversion recovery TSE pre- and postcontrast; GE Healthcare: 3D fast-spoiled gradient-recalled, 3D-T1WI fast-spoiled gradient-recalled, 2D-T2WI double inversion recovery FSE, 2D-T1WI double inversion recovery FSE pre- and postcontrast). More detailed information of the sequences was previously described in the study-design article. ${ }^{24}$

Six observers who were trained in the same institution to delineate plaque components evaluated the MR images of the symptomatic carotid artery with the VesselMass software (Department of Radiology, Leiden University Medical Center). All observers were extensively trained to manually delineate the vessel wall and plaque components on a test set. Subsequently, they had to demonstrate good interobserver agreement for all parameters (interclass correlation coefficient $/ \kappa$ values $\geq 0.6$ ) on a validation set that was delineated by experts with $>15$ years of experience (M.E.K. and A.v.d.L.) before they could start delineating the MR images of the PARISK study.

The observers manually delineated the contours of the inner and outer vessel wall, LRNC, IPH, and calcifications using VesselMass. Then, the software (VesselMass) automatically generated a report with the areas of the lumen, the vessel wall, and each plaque component in each MR imaging section and the total volumes on all MR imaging slices of each plaque component, the lumen, and the vessel wall. The total volumes are the sum of the total area in each section multiplied by the section thickness, taking into account the section gap. The observers were blinded to clinical data and other imaging tests.

A total of $9 \mathrm{MR}$ imaging slices of $2 \mathrm{~mm}$ each covering the entire plaque ( 4 slices in the common carotid artery proximal to bifurcation and 5 slices in the internal carotid artery distal to bifurcation) were included in analysis. The volumes of the lumen, wall (the total volume of the vessel wall including the plaque in $9 \mathrm{MR}$ imaging slices), and total vessel (calculated as lumen volume + wall volume) were used to calculate relative wall volume to total vessel volume (Wall Volume / Total Vessel Volume $\times 100 \%$ ). For each plaque, the presence and volumes of an LRNC, IPH, and calcifications were assessed using multisequence imaging criteria previously validated with histology. ${ }^{27}$ When the LRNC also had internal IPH, we reported the internal IPH as IPH. In addition, the IPH volume was always considered as part of the LRNC. ${ }^{28}$ The relative volume of plaque components to wall volume was calculated (eg, Percentage $\mathrm{IPH}=\mathrm{IPH}$ Volume $/$ Wall Volume $\times 100 \%$ ). Fibrous cap status was divided in 2 categories: thick versus thin-or-ruptured fibrous cap (TRFC) based on previously published criteria. ${ }^{29}$

\section{Statistical Analysis}

Categoric variables are presented as absolute numbers and relative frequencies. Continuous variables are presented as mean \pm $\mathrm{SD}$ or as median with interquartile range (IQR). First, clinical characteristics and plaque parameters in patients with and without an ulceration at baseline and in patients with and without a new ulceration at 2-year follow-up were compared. Continuous variables were compared using a $t$ test or Mann-Whitney $U$ test, and categoric data were evaluated using the $\chi^{2}$ or Fisher exact test.

The association between plaque characteristics and the presence of an ulcer at baseline (cross-sectional analysis) was evaluated using binary logistic regression and reported with ORs and 95\% confidence intervals. The relative volumes of LRNC, IPH, and calcifications were natural log-transformed after adding $1 \%$ to deal with volumes of zero [eg, $\ln (\% \mathrm{IPH}$ Volume +1$)$ ] due to skewed distribution. Because a larger vessel wall volume increased both the risk of having a plaque ulceration and the presence and size of different plaque components, we adjusted the significant associations between plaque composition and plaque ulceration for wall volume. Additional adjustment was performed for all known risk factors (age, sex, body mass index, hypertension, hypercholesterolemia, diabetes mellitus). A sensitivity analysis was performed to test the importance of time intervals among the following: 1) event to MDCTA baseline, 2) event to MR imaging baseline, and 3) MDCTA baseline to MR imaging baseline on the found associations. Because of the relatively low number of new ulcers, only simple statistical testing was performed using the Mann-Whitney $U$ test and Fisher Exact test in the prospective analysis. Plaque parameters were taken as predictors, and the presence of an ulcer, as an outcome variable. A receiver operating characteristic curve and an area under the curve (AUC) were performed to establish the strength of the found associations. $P<.05$ was considered significant (2-sided). All calculations were performed using SPSS, Version 21 (IBM, 2012).

\section{RESULTS}

\section{Patient Characteristics}

From the 240 included patients in the PARISK study, 193 patients underwent both MDCTA and MR imaging at baseline. Thirteen patients were excluded because of poor MDCTA baseline quality $(n=2)$, poor MR imaging quality $(n=5)$, and inadequate MR imaging scan range $(n=6)$. Thus, for cross-sectional analysis, 180 patients were eligible. From these 180 patients, 118 were scheduled for follow-up imaging, but only 75 (64\%) patients underwent MDCTA after 2 years of follow-up. The main reasons that followup imaging was not performed were logistic problems $(n=17)$, contraindications to contrast material $(n=14)$, withdrawal of informed consent $(n=9)$, and death $(n=3)$. An additional 2 patients were excluded because of carotid endarterectomy in the symptomatic carotid artery between the baseline and follow-up scan $(n=1)$ and poor MDCTA follow-up quality. Therefore, 73 patients were eligible for prospective analysis. Figure 1 shows a flow diagram of participants included and excluded from the study.

Clinical characteristics of the included patients at baseline are shown in Table 1 . The median age of the study population was 68 years (range, 63-73 years); $71 \%(127 / 180)$ of the participants were men.

\section{Baseline Imaging Characteristics}

The time interval between the neurologic event and baseline MDCTA was 34 days (IQR, 15-55 days), and the time between the neurologic event and MR imaging, 47 days (IQR, 30-67 


\section{0 symptomatic patients}

From the PARISK study with mild-to-moderate carotid artery stenosis

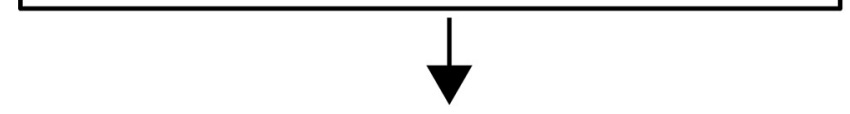

193 patients

Baseline MDCTA and MRI scan present

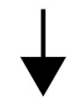

180 patients

Good quality baseline MDCTA and MRI

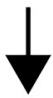

118 patients

Were scheduled for MDCTA follow-up scan

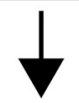

\section{5 patients}

MDCTA follow-up scan present

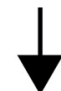

73 patients

Good quality baseline MDCTA and MRI anf follow-up MDCTA
No baseline MDCTA performed $(n=37)$

No MRI baseline performed $(n=8)$

Informed consent withdrawal $(n=2)$

Wrong scan range MRI $(n=6)$

Poor quality MRI baseline $(n=5)$

Poor quality MDCTA baseline $(n=2)$

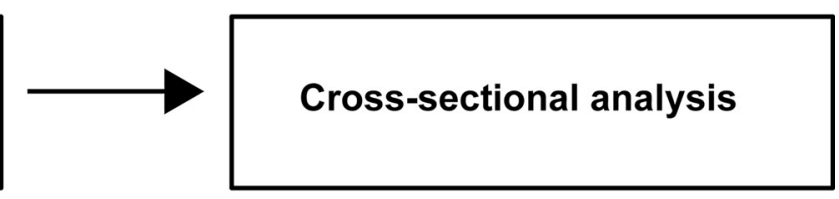

Logistical problems $(n=17)$

Contra-indications for contrast material $(n=14)$

Informed consent withdrawal $(n=9)$

Patient died $(n=3)$

Carotid endarterectomy between scans $(n=1)$

Poor quality MDCTA follow-up

FIG 1. Flow diagram explaining the reasons for exclusion of a number of carotid arteries in the final data analysis.

days). The median time between MDCTA and MR imaging baseline scans was 1 day (IQR, 1-25 days) (Table 1 ).

LRNC and IPH were present in $116(64 \%)$ and 69 (38\%) symptomatic carotid arteries, respectively. Most plaques (162, 90\%) contained calcifications at baseline. A TRFC was present in $77(43 \%)$ of the symptomatic carotid arteries.

\section{Baseline Analysis}

At baseline, 27\% (49/180) of the patients had a plaque ulceration in the symptomatic carotid artery. Imaging characteristics of the carotid arteries with and without plaque ulceration are shown in Table 2. Increased total vessel volume, wall volume, and lumen stenosis were associated with the presence of ulcerations at baseline. LRNC, IPH presence, and TRFC were also associated with ulcerated plaques. Moreover, a higher relative LRNC volume and
IPH volume were associated with ulcerations at baseline. Results of binary logistic regression and receiver operating characteristic analysis for significant associations are shown in Table 2.

After we adjusted for wall volume, LRNC and IPH presence (as dichotomous variable) were no longer significantly related to the presence of plaque ulceration $(\mathrm{OR}=1.5 ; 95 \% \mathrm{CI}, 0.6-3.3$ and $\mathrm{OR}=1.6$; 95\% CI, 0.8-3.4, respectively), but the TRFC remained significantly associated with plaque ulceration at baseline $(\mathrm{OR}=$ 2.4; 95\% CI, 1.2-5). After we adjusted for wall volume, relative LRNC volume and IPH volume remained significantly related to the presence of plaque ulceration $(\mathrm{OR}=1.5 ; 95 \% \mathrm{CI}, 1.1-1.9$ and $\mathrm{OR}=1.5$; 95\% CI, 1.1-2.0, respectively). Additional adjustment for all known risk factors did not change the found associations for relative $\mathrm{LRNC}$ volume and IPH volume $(\mathrm{OR}=1.5 ; 95 \% \mathrm{CI}$, $1.1-1.9$ and $\mathrm{OR}=1.5 ; 95 \% \mathrm{CI}, 1.1-2.1$, respectively). The results 
All Baseline $(n=180) \quad$ Ulcer Baseline Absent $(n=131) \quad$ Ulcer Baseline Present $(n=49) \quad P$ Value

\begin{tabular}{lccc}
\hline Characteristics & & & \\
Age $(\mathrm{yr})$ & $68(63-73)$ & $68(62-73)$ & $69(64-73)$ \\
Sex $(\mathrm{male})$ & $127(71 \%)$ & $122(70 \%)$ & $5(83 \%)$ \\
BMI (kg/m $\left.{ }^{2}\right)$ & $26(24-29)$ & $25(24-29)$ & $27(24-29)$ \\
Classification event & & & .41 \\
TIA & $76(42 \%)$ & $64(49 \%)$ & $12(25 \%)$ \\
Stroke & $85(47 \%)$ & $54(41 \%)$ & $31(63 \%)$ \\
Amaurosis fugax & $19(11 \%)$ & $13(10 \%)$ & $6(12 \%)$ \\
Hypertension & $117(65 \%)$ & $85(65 \%)$ & $32(65 \%)$ \\
Hypercholesterolemia & $140(78 \%)$ & $101(77 \%)$ & $39(80 \%)$ \\
Diabetes mellitus & $42(23 \%)$ & $35(27 \%)$ & $7(14 \%)$ \\
Current smoker & $43(24 \%)$ & $35(27 \%)$ & $8(16 \%)$ \\
Interval event/MDCTA (days) & $34(15-55)$ & $36(16-53)$ & $35(13-59)$ \\
Interval event/MR imaging (days) & $47(30-67)$ & $46(30-67)$ & $48(28-69)$ \\
Interval MDCTA/MR imaging (days) & $1(1-25)$ & $1(1-27)$ & .01 \\
\hline
\end{tabular}

Note:-BMI indicates body mass index.

${ }^{a}$ Data are median (IQR) or No. (\%).

Table 2: Characteristics of plaques with and without an ulcer at baseline

\begin{tabular}{|c|c|c|c|c|}
\hline & $\begin{array}{c}\text { Ulcer Absent }(n=131) \\
\text { Median (IQR) or No. (\%) }\end{array}$ & $\begin{array}{c}\text { Ulcer Present }(n=49) \\
\text { Median (IQR) or No. (\%) }\end{array}$ & $\begin{array}{c}\text { Binary Logistic Regression OR } \\
(95 \% \mathrm{Cl}) \\
\end{array}$ & $\begin{array}{l}\text { ROC Analysis } \\
\text { AUC }(95 \% \mathrm{Cl})\end{array}$ \\
\hline NASCET (\%) & $8(0-29)$ & $21(0-37)$ & $1.01(1.00-1.03)$ & $0.61(0.52-0.71)$ \\
\hline ECST $(\%)$ & $55(45-65)$ & $59(48-70)$ & $1.02(1.00-1.05)$ & \\
\hline Minimal diameter (mm) & $4(3.3-4.9)$ & $3.4(2.8-4.7)$ & $0.06(0.00-1.00)$ & $0.40(0.30-0.50)$ \\
\hline Total vessel volume $\left(\mathrm{cm}^{3}\right)$ & $1.41(1.16-1.64)$ & $1.60(1.29-2.12)$ & $4.9(2.1-11.4)$ & $0.64(0.54-0.74)$ \\
\hline Lumen volume $\left(\mathrm{cm}^{3}\right)$ & $0.56(0.45-0.72)$ & $0.58(0.46-0.80)$ & $2.8(0.6-12.1)$ & \\
\hline Wall volume $\left(\mathrm{cm}^{3}\right)$ & $0.85(0.70-0.98)$ & $1.02(0.77-1.28)$ & $12.1(3.5-42.0)$ & $0.67(0.58-0.77)$ \\
\hline \% Wall volume & $59(53-66)$ & $61(57-68)$ & $1.01(0.99-1.04)$ & \\
\hline LRNC presence & $78(60 \%)$ & $38(78 \%)$ & $2.4(1.1-5.0)$ & $0.59(0.50-0.68)$ \\
\hline \% LRNC volume & $1(0-10)$ & $15(1-31)$ & $1.7(1.3-2.2)$ & $0.70(0.60-0.79)$ \\
\hline IPH presence & $42(32 \%)$ & 27 (55\%) & $2.6(1.3-5.1)$ & $0.61(0.52-0.71)$ \\
\hline \% IPH volume & $0(0-3)$ & $5(0-23)$ & $1.7(1.3-2.2)$ & $0.67(0.56-0.76)$ \\
\hline $\begin{array}{l}\text { Calcifications presence } \\
\text { MR imaging }\end{array}$ & 118 (90\%) & $44(90 \%)$ & $0.97(0.33-2.88)$ & \\
\hline $\begin{array}{l}\text { \% Calcifications volume } \\
\text { MR imaging }\end{array}$ & $5(2-8)$ & $3(1-7)$ & $0.73(0.50-1.07)$ & \\
\hline $\begin{array}{l}\text { Calcification presence } \\
\text { MDCTA }\end{array}$ & 117 (89\%) & $45(92 \%)$ & $0.74(0.23-2.38)$ & \\
\hline $\begin{array}{l}\text { Calcification absolute } \\
\text { volume MDCTA }\left(\mathrm{mm}^{3}\right)\end{array}$ & $31.4(4.3-80.4)$ & $15.8(2.8-51.9)$ & 0.99 (0.999-1.002) & \\
\hline Thin-or-ruptured FC & $45(34 \%)^{a, b}$ & $32(65 \%)$ & $3.4(1.7-6.7)$ & $0.65(0.56-0.74)$ \\
\hline
\end{tabular}

Note:-FC indicates fibrous cap.

${ }^{a}$ Four were missing.

${ }^{b}$ Four had bad quality.

did not change after adjustment for time intervals among the following: 1) event to MDCTA baseline, 2) event to MR imaging baseline, and 3) MDCTA baseline to MR imaging baseline.

\section{Follow-Up Analysis}

A total of 73 patients were eligible for prospective analysis. The time period between the baseline and follow-up MDCTA scans was $25 \pm 2$ months. An ulceration at baseline was present in 19 of the 73 symptomatic plaques (26\%). Most (81\%; 59 of the 73 plaques) of the plaque surfaces remained unchanged: Fifty-one plaques had no ulceration at baseline and follow-up, and 8 plaques had a persistent ulceration at baseline and follow-up. Of the 14 symptomatic plaques that changed during follow-up, 8 ulcers were present at baseline and disappeared after 2 years (Fig 2). In the other 6 plaques (8\%), a new ulcer developed at 2-year follow- up: A new ulceration was seen in 2 plaques with an ulceration at baseline; a new ulceration was seen in a plaque with an ulceration at baseline that disappeared at follow-up; and a new ulceration was seen in 3 plaques without an ulceration at baseline. Figure 2 illustrates an example of a new ulcer development.

All 6 plaques that developed a new ulcer contained LRNC, IPH, calcifications, and a TRFC at baseline. The prevalence of IPH and a TRFC at baseline was significantly higher in plaques with a new ulcer at follow-up than in plaques without one (100\% versus $31 \%$; $P=.002$ and $100 \%$ versus $42 \% ; P=.009$, respectively) (Table 3 ). Plaques that developed a new ulceration had a slightly greater wall volume $\left(1.04 \mathrm{~cm}^{3}\right.$ [IQR, $0.97-1.16 \mathrm{~cm}^{3}$ ] versus $0.86 \mathrm{~cm}^{3}$ [IQR, $\left.\left.0.73-1.00 \mathrm{~cm}^{3}\right] ; P=.029\right)$, a higher relative LRNC volume $(23 \%$ [IQR, 13-31\%] versus $2 \%$ [IQR, $0-14 \%] ; P=.002$ ), and a higher relative IPH volume (14\% [IQR, 8-24\%] versus $0 \%$ [IQR, 0-5\%; 

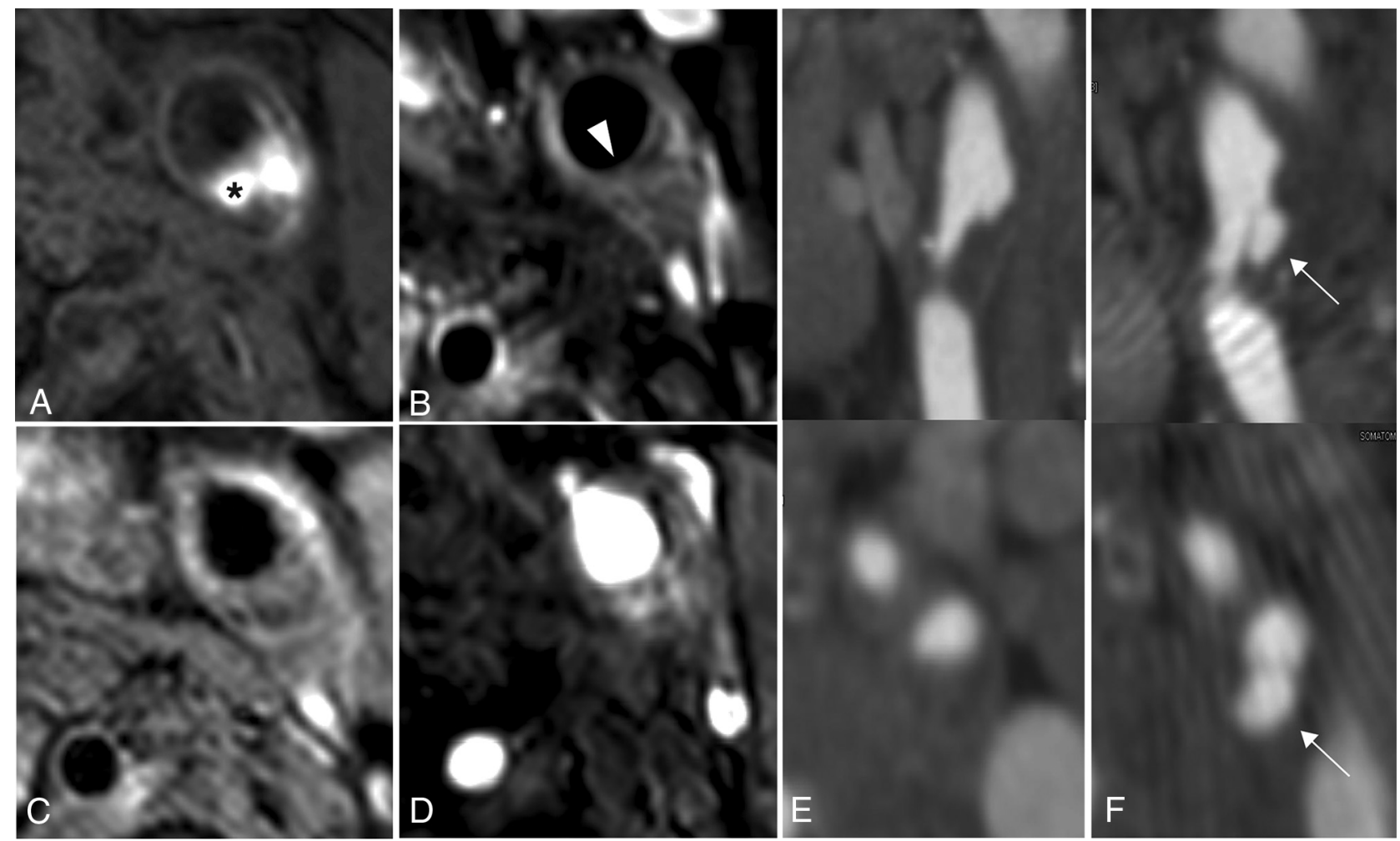

FIG 2. Ulceration development during follow-up and plaque composition at baseline of a 56-year-old female patient. MR imaging of carotid plaque proximal to the ulceration: precontrast T1-weighted turbo field echo sequence $(A)$, postcontrast T1-weighted quadruple inversion recovery TSE sequence $(B)$, T2-weighted TSE sequence $(C)$, time-of-flight sequence $(D)$, and MDCTA of irregular plaque at baseline $(E)$ and of ulceration at 2-year follow-up $(F)$. The asterisk marks the intraplaque hemorrhage. The white arrowhead points to thin-or-ruptured fibrous cap. White arrows point to the ulceration.

Table 3: Characteristics of plaques with and without a new ulceration at 2-year followup $^{a}$

\begin{tabular}{lccc}
\hline & $\begin{array}{c}\text { New Ulceration } \\
\text { Absent }(\boldsymbol{n}=67)\end{array}$ & $\begin{array}{c}\text { New Ulceration } \\
\text { Present }(\boldsymbol{n}=6)\end{array}$ & $\begin{array}{c}\boldsymbol{P} \\
\text { Value }\end{array}$ \\
\hline NASCET (\%) & $16(0-34)$ & $11(0-34)$ & .95 \\
ECST (\%) & $57(47-68)$ & $62(53-71)$ & .45 \\
Minimal diameter $(\mathrm{mm})$ & $3.8(2.8-4.6)$ & $3.4(2.9-4.8)$ & .80 \\
Total vessel volume $\left(\mathrm{cm}^{3}\right)$ & $1.49(1.2-1.75)$ & $1.61(1.53-1.87)$ & .17 \\
Lumen volume $\left(\mathrm{cm}^{3}\right)$ & $0.54(0.44-0.77)$ & $0.59(0.55-0.69)$ & .43 \\
Wall volume $\left(\mathrm{cm}^{3}\right)$ & $0.86(0.73-1.00)$ & $1.04(0.97-1.16)$ & .029 \\
\% Wall volume & $60(52-67)$ & $64(59-67)$ & .37 \\
LRNC presence & $41(61 \%)$ & $6(100 \%)$ & .08 \\
\% LRNC volume & $2(0-14)$ & $23(13-31)$ & .002 \\
IPH presence & $21(31 \%)$ & $6(100 \%)$ & .002 \\
\% IPH volume & $0(0-5)$ & $14(8-24)$ & $<.001$ \\
Calcifications presence MR & $63(94 \%)$ & $6(100 \%)$ & 1.00 \\
$\quad$ imaging & $5(3-8)$ & $6(2-9)$ & .79 \\
\% Calcifications volume MR & & & \\
$\quad$ imaging & $60(90 \%)$ & $5(83 \%)$ & .52 \\
Calcifications presence MDCTA & $32.0(7.0-100.3)$ & $1.9(0.8-91.3)$ & .15 \\
Calcifications, absolute volume & & & \\
$\quad$ MDCTA (mm $\left.{ }^{3}\right)$ & $28(42 \%)$ & $6(100 \%)$ & .009 \\
\hline Thin-or-ruptured FC & & & \\
\hline
\end{tabular}

Note:-FC indicates fibrous cap.

${ }^{a}$ Data are median (IQR) or No. (\%).

$P<0.001$ ) (Table 3). Receiver operating characteristic analysis showed that the wall volume was a fair classifier $(\mathrm{AUC}=0.77$ [95\% CI, 0.66-0.88]), and the relative LRNC and IPH volumes were both strong classifiers for new ulcer development $(\mathrm{AUC}=0.87$ [95\% CI, 0.77-0.97] and AUC $=0.88$ [95\% CI, 0.79-0.97], respectively).

\section{DISCUSSION}

This cross-sectional, prospective, multicenter study of symptomatic patients with mild-to-moderate carotid artery stenosis showed that the presence and development of atherosclerotic plaque ulcerations are associated with large plaque volume and a high content of LRNC and IPH. This finding confirms that plaque composition is an important risk factor for plaque rupture, even in plaques without significant lumen stenosis. The relation between plaque composition and plaque rupture helps to better understand the pathophysiologic pathway from atherosclerosis to a cerebral ischemic event.

Previous studies have mainly focused on the relation between vulnerable plaque components and ischemic events. ${ }^{8,10}$ The current view on the pathophysiology of cerebrovascular ischemic events due to atherosclerosis is 
first the development of plaques with a specific composition, which makes the plaque more vulnerable to plaque rupture. Rupture may result in embolization of plaque material to the intracranial circulation, which occludes the vessel and causes symptoms. More important is the formation of thrombus on a plaque rupture, resulting in embolization of thrombus into the intracranial circulation. Ischemic events are more frequently caused by this pathway than by reduced blood flow or blood pressure due to significant luminal stenosis because most events occur in atherosclerotic carotid disease without severe stenosis. Despite knowledge about this cascade leading to events, the relation between plaque composition and atherosclerotic plaque rupture has hardly been investigated in vivo. Understanding the pathophysiology of atherosclerotic plaque rupture can support the development of preventive strategies of medical treatment that affect the vulnerable components of the plaque and thereby the risk of ischemic stroke. It could also be used to optimize patient selection for carotid endarterectomy on the basis of screening for vulnerable plaque composition.

The results of the current cross-sectional analysis are in line with previous cross-sectional studies. The first study linking plaque ulceration to plaque composition was performed by Lovett et $\mathrm{al}^{21}$ who analyzed carotid artery specimens obtained during endarterectomy and demonstrated that angiographic ulcerations were associated with IPH and a large LRNC. Since then, several cross-sectional studies reported similar associations between plaque composition based on imaging and plaque ulceration. Both Homburg et $\mathrm{al}^{19}{ }^{19}$ and Saba et $\mathrm{al}^{20}$ found, using CT of the plaque, that relative LRNC content was associated with plaque ulceration, whereas calcification proportion was inversely associated with plaque ulceration. A pilot study of the PARISK study demonstrated a relation and colocalization between IPH and disrupted plaque surface. ${ }^{18}$ The current study confirmed these findings and also that wall volume is a strong confounder of the relationship between plaque composition and plaque ulceration. After we adjusted for wall volume, IPH and a large LRNC remained significantly associated with ulceration. However, longitudinal studies are needed to prove a real link between plaque composition and plaque ulceration.

Underhill et $\mathrm{al}^{22}$ found, in a longitudinal study, results similar to ours: a higher percentage wall area, relative LRNC volume, and the presence of LRNC and IPH being predictive of plaque rupture. Nevertheless, our study differs from theirs in some aspects. First, our imaging protocol was different. In contrast to their MR imaging protocol, we used a postcontrast sequence that improves LRNC detection ${ }^{30}$ and a heavily T1weighted TFE sequence, which proved to be optimal for the detection of IPH. ${ }^{31,32}$ We were able to evaluate IPH volumes instead of the presence of IPH only. Second, we used different imaging modalities to establish the presence of ulcerations. Underhill et al used MR imaging and defined plaque rupture as plaque ulceration and/or fibrous cap rupture. We defined plaque rupture as the presence of plaque ulceration only using MDCTA. Wu et $\mathrm{al}^{33}$ demonstrated findings similar to ours by establishing a relationship between carotid artery score, which is mainly a reflection of the extent of LRNC in the plaque, and incident disrupted luminal surface.
Our study has limitations. Because of a relatively small study population that underwent follow-up imaging and a low incidence of ulcerations, multivariable analysis in the longitudinal analysis with adjustment for wall volume could not be performed.

Despite a low ulcer incidence at follow-up, very significant differences were found between plaques with and without a newly developed ulcer. Therefore, we are convinced that meaningful conclusions could be drawn regarding the relation between plaque composition and plaque ulceration risk. Furthermore, it could also be relevant to analyze the atherosclerotic plaque at the asymptomatic contralateral side. However, we did not analyze the asymptomatic artery for 2 reasons. First, the presence of plaque ulcerations at baseline CTA for an asymptomatic artery was low: Only 9\% (17/180) of the asymptomatic arteries visualized by MDCTA had an ulcer at baseline. Moreover, none of the asymptomatic arteries developed a new ulcer after 2-year follow-up. Furthermore, the PARISK study focused the imaging examinations on the symptomatic side. Due to this focus, the contralateral artery was not imaged completely with MR imaging because the alignment of the scan range was performed with the symptomatic artery. This situation resulted in a lower number of asymptomatic arteries eligible for analysis.

A strength of our study is that we combined MDCTA and MR imaging to accurately assess plaque composition with MR imaging and plaque ulceration with MDCTA. Moreover, we used a standard MR imaging range for evaluation of plaque geometry volumes and plaque component volumes. A total of 9 slices was used in the final volume calculations, consisting of the same number of slices in the ICA and the common carotid artery. Therefore, the relative volumes are not biased because of different scan ranges, which may include different lengths of the common carotid artery and ICA. In addition, we noticed that all 6 new ulcerations at follow-up developed within this scan range. Finally, our population had a mild-to-moderate stenosis in contrast to previous studies that mainly focused on severe stenotic arteries, in which carotid endarterectomy was proved to be beneficial. Potentially, patients with mild stenosis and a high-risk plaque may also benefit from carotid endarterectomy, and the number needed to treat in patients with moderate stenosis can probably be improved.

\section{CONCLUSIONS}

The presence and development of plaque ulcerations are associated with large atherosclerotic plaques and a high content of IPH and a LRNC. Identification of predictors for plaque ulceration may prove clinically valuable for preventing plaque rupture and possible recurrent stroke.

\section{ACKNOWLEDGMENTS}

We wish to thank Burhan Hussain for the plaque surface morphology score on MDCTA at baseline.

Disclosures: Kristine Dilba—RELATED: Support for Travel to Meetings for the Study or Other Purposes: Toegepaste en Technische Wetenschappen, grant No. 10813 (Stichting Technische Wetenschappen, Dutch Research Council, the Netherlands).* Mohamed Kassem-RELATED: Grant: The Dutch Research Council, Comments: 
Hestia Impulse. Paul J. Nederkoorn—UNRELATED: GRANTS: Dutch Heart Foundation and Netherlands Health Organisation. M. Eline Kooi-RELATED: Grant: Center for Translational Molecular Medicine PARISK*; UNRELATED: Employment: Maastricht University Medical Center; Grants/Grants Pending: Dutch Research Council, Horizon 2020 Initial Training Networks, Stichting De Weijerhorst*; Travel/Accommodations/ Meeting Expenses Unrelated to Activities Listed: American Heart Association stroke conference, Society for Magnetic Resonance Angiography meeting, Le Fil Rouge meeting, symposium European Society for Cardiology, Comments: I am regularly invited as a speaker, and part of my expenses are reimbursed. ${ }^{*}$ Jolanda J. WentzelRELATED: Grant. Toegepaste en Technische Wetenschappen, Comments: grant for the salary of K. Dilba, grant No. STW 10813.* Anouk C. van Dijk—RELATED: Grant: Center for Translational Molecular Medicine, Dutch Heart Foundation, Comments: This research was performed within the framework of the Center for Translational Molecular Medicine (www.ctmm.nl), project PARISK (Plaque At RISK; grant 01C-202) and supported by the Dutch Heart Foundation.* *Money paid to the institution.

\section{REFERENCES}

1. Petty GW, Brown RD Jr, Whisnant JP, et al. Ischemic stroke subtypes: a population-based study of incidence and risk factors. Stroke 1999;30:2513-16 CrossRef Medline

2. Imbesi SG, Kerber CW. Why do ulcerated atherosclerotic carotid artery plaques embolize? A flow dynamics study. AJNR Am J Neuroradiol 1998;19:761-66 Medline

3. Randomised trial of endarterectomy for recently symptomatic carotid stenosis: final results of the MRC European Carotid Surgery Trial (ECST). Lancet 1998;351:1379-87 Medline

4. Qureshi AI, Alexandrov AV, Tegeler CH, et al; Society of Vascular and Interventional Neurology. Guidelines for screening of extracranial carotid artery disease: a statement for healthcare professionals from the multidisciplinary practice guidelines committee of the American Society of Neuroimaging; cosponsored by the Society of Vascular and Interventional Neurology. J Neuroimaging 2007;17:19-47 CrossRef Medline

5. Rothwell PM, Eliasziw M, Gutnikov SA, et al. Analysis of pooled data from the randomised controlled trials of endarterectomy for symptomatic carotid stenosis. Lancet 2003;361:107-16 CrossRef Medline

6. North American Symptomatic Carotid Endarterectomy Trial Collaborators, et al. Beneficial effect of carotid endarterectomy in symptomatic patients with high-grade carotid stenosis. $\mathrm{N} \mathrm{Engl} \mathrm{J}$ Med 1991;325:445-53 Medline

7. Carotid Surgery Trial: interim results for symptomatic patients with severe (70-99\%) or with mild (0-29\%) carotid stenosisEuropean Carotid Surgery Trialists' Collaborative Group. Lancet 1991;337:1235-43 Medline

8. Zhao H, Zhao X, Liu X, et al. Association of carotid atherosclerotic plaque features with acute ischemic stroke: a magnetic resonance imaging study. Eur J Radiol 2013;82:e465-70 CrossRef Medline

9. Takaya N, Yuan C, Chu B, et al. Association between carotid plaque characteristics and subsequent ischemic cerebrovascular events: a prospective assessment with MRI-initial results. Stroke 2006;37:81823 CrossRef Medline

10. Altaf N, Daniels L, Morgan PS, et al. Detection of intraplaque hemorrhage by magnetic resonance imaging in symptomatic patients with mild to moderate carotid stenosis predicts recurrent neurological events. J Vasc Surg 2008;47:337-42 CrossRef Medline

11. Gupta A, Baradaran H, Schweitzer AD, et al. Carotid plaque MRI and stroke risk: a systematic review and meta-analysis. Stroke 2013;44:3071-77 CrossRef Medline

12. Schindler A, Schinner R, Altaf N, et al. Prediction of stroke risk by detection of hemorrhage in carotid plaques: meta-analysis of individual patient data. JACC Cardiovasc Imaging 2020;13(2 Pt 1):395406 CrossRef Medline

13. Brinjikji W, Rabinstein AA, Lanzino G, et al. Ultrasound characteristics of symptomatic carotid plaques: a systematic review and metaanalysis. Cerebrovasc Dis 2015;40:165-74 CrossRef Medline

14. Eliasziw M, Streifler JY, Fox AJ, et al. Significance of plaque ulceration in symptomatic patients with high-grade carotid stenosis: North American Symptomatic Carotid Endarterectomy Trial. Stroke 1994;25:304-08 CrossRef Medline
15. Cai JM, Hatsukami TS, Ferguson MS, et al. Classification of human carotid atherosclerotic lesions with in vivo multicontrast magnetic resonance imaging. Circulation 2002;106:1368-73 CrossRef Medline

16. Rafailidis V, Chryssogonidis I, Tegos T, et al. Imaging of the ulcerated carotid atherosclerotic plaque: a review of the literature. Insights Imaging 2017;8:213-25 CrossRef Medline

17. Anzidei M, Napoli A, Zaccagna F, et al. Diagnostic accuracy of colour Doppler ultrasonography, CT angiography and blood-pool-enhanced MR angiography in assessing carotid stenosis: a comparative study with DSA in 170 patients (in Italian). Radiol Med 2012;117:54-71 CrossRef Medline

18. van Dijk AC, Truijman MT, Hussain B, et al. Intraplaque hemorrhage and the plaque surface in carotid atherosclerosis: the Plaque At RISK Study (PARISK). AJNR Am J Neuroradiol 2015;36:2127-33 CrossRef Medline

19. Homburg PJ, Rozie S, van Gils MJ, et al. Association between carotid artery plaque ulceration and plaque composition evaluated with multidetector CT angiography. Stroke 2011;42:367-72 CrossRef Medline

20. Saba L, Sanfilippo R, Sannia S, et al. Association between carotid artery plaque volume, composition, and ulceration: a retrospective assessment with MDCT. AJR Am J Roentgenol 2012;199:151-56 CrossRef Medline

21. Lovett JK, Gallagher PJ, Hands LJ, et al. Histological correlates of carotid plaque surface morphology on lumen contrast imaging. Circulation 2004;110:2190-97 CrossRef Medline

22. Underhill HR, Yuan C, Yarnykh VL, et al. Predictors of surface disruption with MR imaging in asymptomatic carotid artery stenosis. AJNR Am J Neuroradiol 2010;31:487-93 CrossRef Medline

23. Xu D, Hippe DS, Underhill HR, et al. Prediction of high-risk plaque development and plaque progression with the carotid atherosclerosis score. JACC Cardiovasc Imaging 2014;7:366-73 CrossRef Medline

24. Truijman MT, Kooi ME, van Dijk AC, et al. Plaque At RISK (PARISK): prospective multicenter study to improve diagnosis of high-risk carotid plaques. Int J Stroke 2014;9:747-54 CrossRef Medline

25. North American Symptomatic Carotid Endarterectomy Trial: methods, patient characteristics, and progress. Stroke 1991;22:71120 CrossRef Medline

26. de Weert TT, Cakir H, Rozie S, et al. Intracranial internal carotid artery calcifications: association with vascular risk factors and ischemic cerebrovascular disease. AJNR Am J Neuroradiol 2009;30:17784 CrossRef Medline

27. Kwee RM, Teule GJ, van Oostenbrugge RJ, et al. Multimodality imaging of carotid artery plaques: 18F-fluoro-2-deoxyglucose positron emission tomography, computed tomography, and magnetic resonance imaging. Stroke 2009;40:3718-24 CrossRef Medline

28. Cappendijk VC, Heeneman S, Kessels AG, et al. Comparison of single-sequence T1WI TFE MRI with multisequence MRI for the quantification of lipid-rich necrotic core in atherosclerotic plaque. J Magn Reson Imaging 2008;27:1347-55 CrossRef Medline

29. Kwee RM, van Engelshoven JM, Mess WH, et al. Reproducibility of fibrous cap status assessment of carotid artery plaques by contrastenhanced MRI. Stroke 2009;40:3017-21 CrossRef Medline

30. Takaya N, Cai J, Ferguson MS, et al. Intra- and interreader reproducibility of magnetic resonance imaging for quantifying the lipidrich necrotic core is improved with gadolinium contrast enhancement. J Magn Reson Imaging 2006;24:203-10 CrossRef Medline

31. Cappendijk VC, Cleutjens $\mathrm{KB}$, Heeneman $\mathrm{S}$, et al. In vivo detection of hemorrhage in human atherosclerotic plaques with magnetic resonance imaging. J Magn Reson Imaging 2004;20:105-10 CrossRef Medline

32. Bitar R, Moody AR, Leung G, et al. In vivo 3D high-spatial-resolution MR imaging of intraplaque hemorrhage. Radiology 2008;249:259-67 CrossRef Medline

33. Wu Z, Yang C, Tang D. In vivo serial MRI-based models and statistical methods to quantify sensitivity and specificity of mechanical predictors for carotid plaque rupture: location and beyond. $J$ Biomech Eng 2011;133:064503 CrossRef Medline 